\title{
Genome-wide characterization of the auxin response factor (ARF) gene family of litchi (Litchi chinensis Sonn.): phylogenetic analysis, miRNA regulation and expression changes during fruit abscission
}

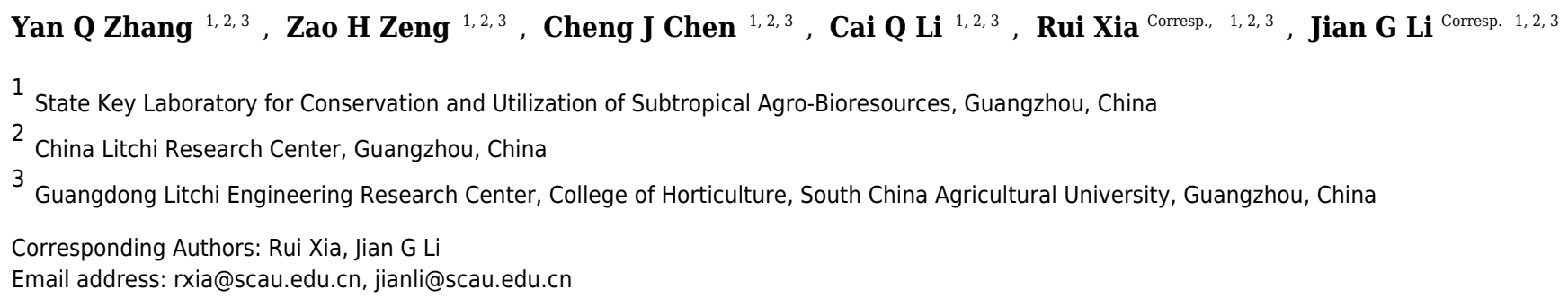

Auxin response factors (ARFs) play fundamental roles in modulating various biological processes including fruit development and abscission via regulating the expression of auxin response genes. Currently, little is known about roles of ARFs in litchi (Litchi chinensis Sonn.), an economical important subtropical fruit tree whose production is suffering from fruit abscission. In this study, a genome-wide analysis of ARFs was conducted for litchi, thirty-nine ARF genes (LCARFs) were identified. Conserved domain analysis showed that all the LCARFs identified have the signature B3 DNA-binding (B3) and ARF (Aux_rep) domains, with only 23 members having the dimerization domain (Aux_IAA). Number of exons in LCARF genes ranges from 2 to 16, suggesting a large variation for the gene structure of LCARFs. Phylogenetic analysis showed that the 39 LcARFs could be divided into three main groups: class I, II, III. Totally, twenty-three LCARFs were found to be potential targets of small RNAs, with three conserved and one novel miRNA-ARF (miRN43-ARF9) regulatory pathways discovered in litchi. Expression patterns were used to evaluate candidate LCARFs involved in various developmental processes, especially in flower formation and organ abscission. The results revealed that most ARF genes likely acted as repressors in litchi fruit abscission, i.e. $A R F 2 D / 2 E, 7 A / 7 B, 9 A / 9 B, 16 A / 16 B$, while a few $L C A R F s$, such as $L C A R F 5 A / B$, might positively involve in this process. These findings provide useful information and resources for further studies on the roles of ARF genes in litchi growth and development, especially in the process of fruit abscission. 
1 Genome-wide characterization of the auxin response

2 factor (ARF) gene family of litchi (Litchi chinensis

3 Sonn.): phylogenetic analysis, miRNA regulation and

4 expression changes during fruit abscission

6 Yanqing Zhang ${ }^{1,2,3}$, Zaohai Zeng ${ }^{1,2,3}$, Chengjie Chen ${ }^{1,2,3}$, Caiqin Li $^{1,2,3}$, Rui Xia ${ }^{1,2,3 *}$, Jianguo $7 \mathrm{Li}^{1,2,3 *}$

$8{ }^{1}$ State Key Laboratory for Conservation and Utilization of Subtropical Agro-Bioresources

$9{ }^{2}$ China Litchi Research Center, Guangzhou, China

$10{ }^{3}$ Guangdong Litchi Engineering Research Center, College of Horticulture, South China

11 Agricultural University, Guangzhou, China

13 Corresponding Author:

14 Rui Xia ${ }^{1,2,3}$

15 Yuehan Road 483, Wushan, Tianhe, Guangzhou, Guangdong Province, 510642, China

16 Email address: rxia@scau.edu.cn

17 Jianguo $\mathrm{Li}^{1,2,3}$

18 Yuehan Road 483, Wushan, Tianhe Guangzhou, Guangdong Province, 510642, China

19 Email address: jianli@scau.edu.cn 


\section{Abstract}

23 Auxin response factors (ARFs) play fundamental roles in modulating various biological

24 processes including fruit development and abscission via regulating the expression of auxin

25 response genes. Currently, little is known about roles of ARFs in litchi (Litchi chinensis Sonn.),

26 an economical important subtropical fruit tree whose production is suffering from fruit

27 abscission. In this study, a genome-wide analysis of ARFs was conducted for litchi, thirty-nine

28 ARF genes (LcARFs) were identified. Conserved domain analysis showed that all the LcARFs

29 identified have the signature B3 DNA-binding (B3) and ARF (Aux_rep) domains, with only 23

30 members having the dimerization domain (Aux_IAA). Number of exons in LcARF genes ranges

31 from 2 to 16, suggesting a large variation for the gene structure of $L c A R F s$. Phylogenetic

32 analysis showed that the 39 LcARFs could be divided into three main groups: class I, II, III.

33 Totally, twenty-three $L c A R F s$ were found to be potential targets of small RNAs, with three

34 conserved and one novel miRNA-ARF (miRN43-ARF9) regulatory pathways discovered in

35 litchi. Expression patterns were used to evaluate candidate $L c A R F s$ involved in various

36 developmental processes, especially in flower formation and organ abscission. The results

37 revealed that most ARF genes likely acted as repressors in litchi fruit abscission, i.e. $A R F 2 D / 2 E$,

$387 A / 7 B, 9 A / 9 B, 16 A / 16 B$, while a few $L c A R F s$, such as $L c A R F 5 A / B$, might positively involve in

39 this process. These findings provide useful information and resources for further studies on the

40 roles of ARF genes in litchi growth and development, especially in the process of fruit

41 abscission. 


\section{Introduction}

44 Auxin plays a central role in numerous aspects of plant developmental and physiological 45 processes, including embryogenesis, apical dominance, vascular elongation, flowering, fruit 46 development, and lateral root initiation (Woodward \& Bartel, 2005; Fleming, 2006). Auxin 47 response factors (ARFs) are a group of important transcription factors in the auxin signaling 48 pathway, which can activate or repress the expression of early/primary auxin response genes by 49 binding to the auxin response element (AuxRE) site in their promoter regions (Liscum \& Reed, 50 2002; Guilfoyle \& Hagen, 2007). A typical ARF is characterized by a highly conserved N51 terminal B3-type DNA binding domain (DBD) that recognized the AuxRE motif, an activation 52 domain $(\mathrm{AD})$ or repression domain $(\mathrm{RD})$, and a carboxy-terminal dimerization domain (CTD: 53 domain III/IV), which is involved in protein-protein interactions by dimerizing with 54 auxin/indole-3-acetic acid (Aux/IAA) family genes (Kim, Harter \& Theologis, 1997; Guilfoyle 55 \& Hagen, 2007; Piya et al., 2014).

ARFs exert pivotal function in the regulation of plant growth and development through the auxin 58 signaling pathway ( Kepinski and Leyser, 2005; Li et al., 2016). Due to the significance of ARFs, genome-wide characterization of ARFs have been completed in many species such as model plants Arabidopsis (Hagen \& Guilfoyle, 2002) and Solanum lycopersicum (Zouine et al., 2014), important crops such as soybean (Ha et al., 2013; Le et al., 2016) and maize (Xing et al., 2011; Wang et al., 2012), fruit trees like citrus (Citrus sinensis) (Xie et al., 2015) and apple (Malus domestica) (LUO et al., 2014), and so on. From embryogenesis to flowering, mutants in members of ARFs exhibit diverse phenotypes, which show their unique and redundant functions for plant development. For instance, in Arabidopsis thaliana, arf1 and arf2 mutations affect leaf senescence and floral organ abscission (Ellis et al., 2005) and the loss of AtARF3 causes defectives in gynoecium patterning (Nemhauser, Feldman \& Zambryski, 2000; Liu et al., 2014a). AtARF5 influences embryo, root and shoot development (Krogan et al., 2012; Crawford et al., 2015). AtARF9 acts in suspensor cells to mediate hypophysis specification (Rademacher et al., 2012), and AtARF10/16/17 play vital roles in negatively regulating seed germination and postgermination activities (Liu et al., 2007).

Recently, small RNAs, especially miRNAs, have been emerging as critical regulators in almost all aspects of plant growth and development. Many members of the ARF family have been reported to be targets of miRNAs. In Arabidopsis, AtARF6 and AtARF8 are targets of miR167 76 (Wu, Tian \& Reed, 2006), AtARF10/16/17 are targets of miR160 (Liu et al., 2007, 2010), and 
$77 A R F 2 / 3 / 4$ are targets of trans-acting siRNAs (tasiRNAs) generated from miR390-targeted TAS3

78 gene (trans-acting siRNA gene 3) (Allen et al., 2005; Axtell et al., 2006). These targeting

79 relationships of miRNA on ARF genes are widely conserved in land plants (Xia, Xu \& Meyers,

80 2017) and also in horticultural plants (Chen et al., 2018b). It has been reported that down-

81 regulation of $A R F 6$ and $A R F 8$ by miR167 leads to floral development defects and female sterility

82 in tomatoes (Liu et al., 2014b). Down-regulation of sly-miR160, increasing the expression of its

83 targets SlARF10/16/17, regulates auxin-mediated ovary patterning as well as floral organ

84 abscission and lateral organ lamina outgrowth (Damodharan, Zhao \& Arazi, 2016). In

85 Arabidopsis and tomato, the tasiRNA-mediated regulation of $A R F 2$ involved in controlling the

86 onset of leaf senescence and floral organ abscission (Ellis et al., 2005; Lim et al., 2010; Guan et

87 al., 2014; Ren et al., 2017).

88

89 Litchi (Litchi chinensis sonn.), an important economic fruit trees in southern China, usually

90 undergo serious fruit abscission before harvest, leading to a low yield. Many studies demonstrate

91 ARFs play critical roles in regulating plant organ abscission (Ellis et al., 2005; Kuang et al.,

92 2012; Guan et al., 2014; Xie et al., 2015; Xu et al., 2015), while which ARFs are involved or

93 more important than the other in the fruit abscission in litchi remains elusive. Here, we identified

9439 ARF genes in litchi. Gene structure, phylogeny and targeting relationship with miRNAs were

95 characterized. The expression of $L c A R F s$ was examined in diverse tissues and in the process of

96 fruit abscission which was induced by three different treatments. Among them, $A R F 2 D / 2 E$,

$977 A / 7 B, 9 A / 9 B, 16 A / 16 B$ and $L c A R F 5 A / B$, were found to be associated with litchi fruit abscission.

98 Our results offer new knowledge and resources to study the function of plant ARF genes and

99 their roles in the fruit abscission in litchi. 


\section{Materials \& Methods}

\section{Plant materials and treatments}

103 The young fruitlet used for RNA-seq in our study were collected from 9-year-old litchi trees

104 (Litchi. chinensis Sonn. cv. 'Feizixiao') in an orchard located at South China Agricultural

105 University (Guangzhou, China). Three treatments have been performed 25 day after anthesis.

106 The three treatments included ethephon (ETH), girdling plus defoliation (GPD) and dipping in

107 20mg/L 2, 4-D for one minute after girdling plus defoliation (GPDD). Details can refer to Li et al

108 (Li et al., 2015) and Peng et al (Peng et al., 2013). Samples of 'Feizixiao' (FZX) for qRT-PCR

109 were obtained from the orchard of Guangzhou Fruit Research Institute (Guangdong, China).

110 Tissues including fruit-bearing shoots (FBS), young leaves (YL), mature leaves (ML), female

111 flower (FF), male flower (MF), sex undeterminated flower (USF) and young fruit (YF) of 25

112 days after fertilization were collected from different directions of each tree. After separation, all

113 tissues were quickly frozen in liquid nitrogen and stored at $-80^{\circ} \mathrm{C}$ before usage.

114

\section{Identification and phylogenetic analyses of LcARFs}

116 Amino acid sequences of 25 and 23 ARFs from rice (Wang et al., 2007) and Arabidopsis

117 (Okushima et al., 2005; Wang et al., 2007) were downloaded from UniProt

118 (http://www.uniprot.org/) and 22 ARFs from tomato (Zouine et al., 2014) were downloaded from

119 Sol Genomics Network (https://solgenomics.net/). These ARFs were used as bait to identify

120 potential ARFs in litchi genome and obtained by Blast analysis in TBtools (Chen et al., 2018a),

121 with the E-value $1 \mathrm{e}^{-10}$. All sequences were then further validated by conserved domain search

122 against the CDD (http://www.ncbi.nlm.nih.gov/cdd/) and PFAM (http://pfam.xfam.org/)

123 databases. Based on the optimized alignment of amino acid sequences of LcARFs proteins,

124 achieved by TrimAL 1.3 (http://phylemon2.bioinfo.cipf.es/index.html) after the initial sequence

125 alignment in ClustalX 2.1, a maximum likelihood (ML) tree was constructed in MEGA 7.0

126 software with a bootstrap of 1000 replicates. Intron and exon distribution patterns and genome

127 structure were analyzed and visualized in TBtools (Chen et al., 2018a). The detail of predicted

128 protein sequences of LcARFs were shown in Data S1 and S2.

129

\section{$130 \boldsymbol{L C A R F}$ targets of miRNAs and RNA-seq analysis}

131 Most of the $A R F$-targeting miRNA/tasiRNAs were identified in our previous study (Ma et al.,

132 2018). To further validate the targeting relationship, target sites were verified by psRNATarget

133 (http://plantgrn.noble.org/psRNATarget) (Dai \& Zhao, 2011) and miRBase

134 (http://www.mirbase.org/). 
135 RNA-seq analysis was carried on as described previously (Li et al., 2013). These RNA-seq data 136 was normalized using TPM method (Wagner, Kin \& Lynch, 2012). Based on the sequences of

137 the identified $L c A R F s$, a BLASTN search was conducted to find their gene counterparts in 138 previous data ( $\mathrm{Li}$ et al., 2013). Finally, a global gene expression profiles were visualized by

139 heatmap via TBtools software (Chen et al., 2018a).

140

\section{Quantitative RT-PCR}

142 Total RNA of above seven tissue samples was extracted by column plant RNAout kit (TIANDZ,

143 China) according to the manufacturer's instructions. About $2 \mu \mathrm{g}$ total RNA was applied to

144 synthesized first strand cDNA using reverse transcriptase AT311-03 (TRANSGEN BIOTECH,

145 China). PCR primers (Table S1) of $L c A R F$ s and reference genes GAPDH and EF (Zhong et al.,

146 2011) were designed by Primer Premier 5.0. qRT-PCR was performed according to the

147 manufacturer's specifications of THUNDERBIRD qPCR MIX QPS-201 (TOYOBO, China) on a

148 LightCycler 480 (Roche, Switzerland). Each expression profile was independently verified in

149 three biological replicates. Relative expression level of each gene was calculated by the $2^{-\Delta \Delta \mathrm{Ct}}$

150 method (Livak \& Schmittgen, 2001). Significance analysis was conducted in SPSS vision 22.0

151 and visulized by SigmaPlot 12.5 . 


\section{Results}

\section{Identification and phylogenetic analysis of LcARF genes}

155 To identify all ARF members in litchi, protein sequences of ARFs in rice, Arabidopsis and

156 tomato were used as queries in BLASTP to search against litchi annotated gene database. Sixty-

157 eight potential ARF genes were identified. After redundant result elimination and further

158 conserved domain validation, 39 LcARFs were obtained ultimately. Among them, 23 protein

159 sequences contained B3, ARF and the Aux/IAA domains. Length of these litchi ARF proteins

160 ranged from 260 (LcARF1B) to 1200 (LcARF4A) and the relative molecular mass of them

161 varied from 29522.5 (LcARF1B) to $144936.9 \mathrm{Da}$ (LcARF16C), with PIs in the range of 5.28

162 (LcARF16C) to 8.64 (LcARF19A) (Table S2). According to the subcellular localization

163 predictor ( $\underline{\text { CELLO v.2.5; http://cello.life.nctu.edu.tw) }}$ prediction, most LcARFs were predicted to

164 be located in the nucleus.

166 A phylogenetic tree was generated using the maximum likelihood method based on alignment of

167 litchi ARFs with their orthologs from rice, Arabidopsis and tomato (Fig. 1). All 109 ARFs fell

168 into three broad groups: class I, II and III, which contained 53, 34 and 22 members, respectively.

169 Obviously, most ARFs of the four species were clustered together in the first two classes. Litchi

170 ARFs were named according to their positions with orthologs from the other three species in the

171 tree. Thus, the $39 \mathrm{LcARFs}$ could also be assigned to three separate clusters as well. Class I

172 contained LcARF1/2/3/4/9/18 (1A/B/C/D, 2A/B/C/D/E/F, 3A/B, 4A/B/C, 9A/B, 18A/B); Class

173 II included LcARF5/6/7/8/19 (5A/B, 6A/B/C/D, 7A/B, 8A/B, 19A/B); Class III included

174 LcARF10/16/17 (10A/B, 16A/B/C/D, 17A/B).

175

\section{Gene structure and conserved domains of litchi ARFs}

177 To better understand the structure evolution of LcARF genes, their gene structure (intron/exon

178 number and positions) and functional domains were analyzed. As shown in Fig. 2, 23 LcARFs

179 harbored the typical ARF protein structure which composed of a highly conserved DNA-binding

180 domain (DBD) in the N-terminal region with a plant specific B3-type subdomain, an Auxin-resp

181 subdomain, and an AUX_IAA dimerization subdomains. The remaining 16 LcARFs contained

182 only B3 and Auxin_resp subdomains. Gene structure analysis revealed that the exon number of

183 class III (2-4 exons) was significantly less than the other two groups (8-17 exons for class II and

184 10-16 exons for class I). These results provided additional evidence confirming the phylogenic

185 relationships among LcARFs.

186 


\section{Analyses of miRNA targeting $L c \boldsymbol{A R F s}$}

188 Twenty-two out of the 39 LcARF genes were found to be the targets of miRNA (Fig. 3). All 189 group members of class III ( $L C A R F 10 A / B, 16 A / B / C / D$ and $17 A / B$ ) were found to be targeted by

190 Lc-miR160. $L c A R F 6 A / B / C / D$ and $8 A / B$ were members of class II and all of them were

191 collectively targeted by Lc-miR167. Two kinds of miRNA targeting patterns were observed in

192 the class I. One group comprising $L c A R F 2 E / 2 F / 4 B / 4 C / 3 A / 3 B$ were targeted by tasiARFs. In

193 litchi, LcTAS3 are divided into two subgroups, long TAS3 genes(LcTAS3_1 and LcTAS3_2 ) and

194 short TAS3 genes (LcTAS3_3 and LcTAS3_4 ), which trigger to produce two or one tasiARFs

195 when cleaved by miR390 (Ma et al., 2018). Additionally, $L c A R F 2 E / 2 F$ incorporated one target

196 site of tasiARF, while $L c A R F 3 A / 3 B / 4 B / 4 C$ contained two, which is in accordance with our

197 previous study (Xia, Xu \& Meyers, 2017). Interestingly, in the other group, a novel miRNA- $A R F$

198 pathway was discovered, in which $L c A R F 9 A / B$ was targeted by the Lc-miRN43.

199

200

\section{Expression of LcARF Genes in different organs and tissues}

201

A large body of evidence support the importance of ARF genes in plant growth and development

202 (Ellis et al., 2005; Guilfoyle \& Hagen, 2007; Lim et al., 2010; Li et al., 2016). To explore how LcARF genes function in the development of litchi, we examined their expression levels in various litchi organs/tissues by qRT-PCR. Seven organs/tissues samples, composing fruitbearing shoots (FBS), male flower (MF), female flower (FF), sex undeterminated flower (USF), mature leaves (ML), young leaves (YL) and young fruits (YF) in 'FZX', were collected and tested. As shown in Fig. 4A, expression of all $A R F S$ was detected at least in several tissues. Generally, most LcARFs show low expression in YL and YF but high in USF. LcARFs from different clades showed various expression patterns. $L c A R F s$ from class I and III were detected

211 these $L c A R F s$ in different classes may perform different functions in mature leaves development

212 (Fig. 4A). Additionally, as is shown in Fig. 4B, ten LcARFs (LcARF2B, 3A, 4A/B, 5A/B, 6D,

$2139 A / B, 18 B)$ were significantly higher expressed in FF than MF, implying their potential function

214 in ovule and ovary derived from female flowers. Remarkably, three LcARFs (LcARF8A,

$215 L C A R F 10 A / B$ ) were of significantly higher expression in MF than FF, indicated that they might

216 play roles in male flower formation. Additionally, a table for LcARFs and their potential

217 function was concluced in Data S3 according to early study which may be useful for further

218 functional validation.

219

220

\section{Expression profiles of LcARF genes in response to ETH, GPD, and GPDD treatment}


221 Auxin is a critical signal in the abscission of fruits in plants (Blanusa et al., 2005; Meir et al., 222 2010; Xie et al., 2013). To explore the role of $L c A R F s$ in fruitlet abscission in litchi, RNA-seq 223 was carried on and transcription levels of LcARF genes in the abscission zone were investigated 224 under three treatments (ETH, GPD, GPDD). Both the ETH and GPD treatments promote fruitlet 225 abscission while GPDD delays the process (Peng et al., 2013; Li et al., 2015). As the results in 226 Fig. 5, the transcript expression of most $L c A R F s$ were decreased after treated by ETH and GPD, 227 but upsurge after GPDD treatment, which was corresponding to the process of promoting or 228 inhibiting of fruitlet shedding, respectively. It seemed that there was a negative correlation 229 between $L c A R F s$ and the fruit abscission and among these $L c A R F s$, those from two groups 230 (group 2 and group 3) were particularly representative. Moreover, LcARFs from group 3 were 231 more sensitive to GPDD treatment along with stronger expression than those from group 2 . Thus, 232 we could deduce that $L c A R F s$ from group 3, including $A R F 2 D / 2 E, 7 A / 7 B, 9 A / 9 B, 16 A / 16 B$, 233 played major roles on the litchi fruitlet abscission. In contrast, a few $L c A R F s$, such as group 1 234 including $L c A R F 5 A / 5 B$, showed opposite expression pattern, suggesting that they might function 235 to accelerate the process of abscission. There were as well some LcARFs with no significant 236 expression change and seemed to be unrelated to fruitlet abscission. 


\section{Discussions}

239 Litchi is an important tropic fruit trees and massive fruit abscission before harvest usually leads

240 to low and even no production. Auxin is proposed to be one of the endogenous hormones playing

241 significant roles in the regulation of fruit abscission in litchi (Yuan, 1988; Stern \& Gazit, 2000).

242 In this study, 39 LcARF genes were identified, which was more than the other model plants,

243 such as Arabidopsis (23) (Okushima et al., 2005; Wang et al., 2007), rice (25) (Wang et al.,

244 2007) and tomato (22) (Zouine et al., 2014), implying extensive duplication and diversification

245 of the ARF gene family in litchi. Analysis of conserved motifs revealed that all LcARFs had a

246 typical DBD domain required for efficient binding to AuxRE and a Auxin_resp (Fig. 2) (Hagen

247 \& Guilfoyle, 2002; Ha et al., 2013). However, only 23 of 39 LcARFs contain AUX_IAA

248 domain, which can mediate the dimerization of ARFs or ARF and Aux/IAA protein (Guilfoyle \&

249 Hagen, 2007). Lack of the AUX_IAA domain for dimerization makes it interesting to address

250 questions like how these ARFs function and whether they need dimerization with other proteins.

251 In plants, ARFs can function as transcription activators (ADs) or repressors (RDs), according to

252 the amino acid composition of Auxin_resp domain (Guilfoyle \& Hagen, 2007). In Arabidopsis

253 thaliana, ARF ADs and RDs were proposed to contain biased amino acid sequences, which ARF

254 ADs were enriched in glutamine $(\mathrm{Q})$, while RDs were enriched in serine (S), serine and proline

255 (SP), serine glycine (SG) (Guilfoyle \& Hagen, 2001; Tiwari, Hagen \& Guilfoyle, 2003).

256 Intriguingly, no ARFs in litchi were enriched in Q, but with SPL and SP/SG enrichment; then

257 none of LcARF proteins seems to be an activator (reviewed in (Guilfoyle \& Hagen, 2007)).

258 Further experiments are needed to verify this observation.

259

260 Much evidence demonstrates that miRNAs play essential roles in post-transcriptional gene

261 regulation in plants (Jones-Rhoades, Bartel \& Bartel, 2006; Li \& Zhang, 2016). It has been found

262 that several ARF genes are regulated by a few miRNAs. In our work, 22 out of 39 LcARF genes

263 were found to be targets of miRNAs (Fig. 3) and $L C A R F$ s from different classes were displayed

264 different miRNA targeting pattern. Members of class III ( $L C A R F 10 A / B, 16 A / B / C / D$ and $17 A / B)$

265 were found to be targeted by Lc-miR160, which might affect flower development of litchi, as

266 down-regulation of $A R F 10 / 16 / 17$ by miRNA160 is reported to regulate floral organ abscission in

267 tomato (Damodharan, Zhao \& Arazi, 2016). Consistent with Arabidopsis, LcARF6/8 were

268 collectively targeted by Lc-miR167. Interestingly, even though there has been reported LCARF8B

269 to be targeted by Lc-miR167 (Ma et al., 2018), LcARF8A was a novel target by Lc-miR167.

270 Notably, in another group, a novel miRNA-ARF pathway was discovered in litchi, in which

$271 L c A R F 9 A / B$ was targeted by Lc-miRN43. In fact, miRN43 is an innovative miRNA as well, for it 
272 is unable to find any ortholog in the database of miRase, which may provide a new idea for us to

273 study the specific functions of litchi.

274

275 The expression profiles can help us screen for candidate $L c A R F$ genes with potentially distinct

276 functions. Most $L c A R F s$ were higher accumulated in mature leaves than young leaves including

$277 L c A R F 2$, and its Arabidopsis homolog AtARF2 has been reported to regulate leaf senescence

278 with high expression in mature leaves (Ellis et al., 2005). Thus we can deduce that LcARF2 is

279 likely to involve in leaf senescence in litchi. It has been reported that Arabidopsis ARF genes

280 regulate flower formation, especially gametophyte development, which are critical for sexual

281 reproduction. AtARF2-AtARF4 and AtARF5 play significant roles in regulating both female

282 and male gametophyte development in Arabidopsis (Liu et al., 2017) and ARF6 and ARF8

283 regulate both stamen and gynoecium maturation (Nagpal, 2005; Ru et al., 2006; Wu, Tian \& Reed, 2006; Liu et al., 2014b). Thus their litchi homologs LcARF2B, 3A, 4A/B, 5A/B, 6D and

285

286

287

288

289

290

291

292

293

294

295

296

297

298

299

300

301

302

303

$8 \mathrm{~A}$ may be important for female and male gametophyte development in litchi, along with significantly higher accumulation in female or male flower. Moreover, LcARF4, 5 and 9 were prominent higher expressed in female flower than male flower, implying their potential function in ovule and ovary development.

ARF genes have been reported to be involved in plant organ abscission. Overexpression of SlARF2 in tomato results in flower organ senescence (Ren et al., 2017) and SlARF1, 2, 7, 11 and 19 showed overlapping functions in tomato abscission (Guan et al., 2014). Similar roles of ARFs in abscission were observed in Arabidopsis (Ellis et al., 2005). Our previous studies show that the treatment of girdling plus defoliation (GPD) in litchi could reduce the transcript level of auxin response factor ( $L c A R F 1)$ mRNA, along with the increase of fruitlet abscission (Kuang et al., 2012). Here in our RNA-seq survey of ARF genes expression, we found that most of the ARF genes show opposite correlation with the fruit abscission, i.e., ARF genes were downregulated by abscission induced treatments (ETH and GPD), but up-regulated by GPDD, in which the abscission of GPD was inhibited by the addition of 2, 4-D, suggesting that the majority of LcARF genes are negatively involved in litchi fruit abscission, especially $L c A R F s$ from group $3(A R F 2 D / 2 E, 7 A / 7 B, 9 A / 9 B, 16 A / 16 B)$, with more prominent expression after GPDD treatment. By contrast, a few $L c A R F s$, such $L c A R F 5 A / 5 B$, show positive correlation with fruit abscission,

304

305

indicating that they might serve as contributing factors to fruit shedding.

\section{Conclusions}


306 In this study, a total of 39 ARF genes were identified from the litchi genome. Comprehensive

307 analyses, including phylogenetic relationship, exon-intron structure, conserved domain, and

308 potential targets for small RNAs, revealed that the ARF gene family was expanded in litchi with

309 species-specific features. A novel miRNA- $A R F$ (miRN43-ARF9) regulatory pathway was

310 discovered, which is likely specific in litchi. Expression profiles in various organs and under

311 different abscission-related treatments (ETH, GPD and GPDD) uncovered the expression

312 diversity of these ARF genes in litchi. Some ARF genes, including $A R F 2 D / 2 E, 7 A / 7 B, 9 A / 9 B$,

$31316 A / 16 B$ and $5 A / 5 B$, likely play predominant roles in the process of litchi fruit abscission. These

314 findings provide new knowledge and resources for further functional characterization of ARF

315 genes in litchi.

316 


\section{References:}

318 Addicott FT, Lynch RS. 1955. Physiology of abscission. Annual Review of Plant Physiology and

319 Plant Molecular Biology 6:211-238. DOI: 10.1146/annurev.pp.06.060155.001235.

320 Allen E, Xie Z, Gustafson AM, Carrington JC. 2005. microRNA-directed phasing during trans-

321 acting siRNA biogenesis in plants. Cell 121:207-221. DOI: 10.1016/j.cell.2005.04.004.

322 Axtell MJ, Jan C, Rajagopalan R, Bartel DP. 2006. A Two-Hit Trigger for siRNA Biogenesis in

323 Plants. Cell 127:565-577. DOI: 10.1016/j.cell.2006.09.032.

324 Blanusa T, Else MA, Atkinson CJ, Davies WJ. 2005. The regulation of sweet cherry fruit

325 abscission by polar auxin transport. Plant Growth Regulation 45:189-198. DOI:

326 10.1007/s10725-005-3568-9.

327 Chen C, Xia R, Chen H, He Y. 2018a. TBtools, a Toolkit for Biologists integrating various HTS-

328 data handling tools with a user-friendly interface. bioRxiv:289660. DOI: 10.1101/289660.

329 Chen C, Zeng Z, Liu Z, Xia R. 2018b. Small RNAs, emerging regulators critical for the

330 development of horticultural traits. Horticulture Research 5:6-8. DOI: 10.1038/s41438-018-

$331 \quad 0072-8$.

332 Crawford BCW, Sewell J, Golembeski G, Roshan C, Long JA, Yanofsky1 MF. 2015. Genetic

333 control of distal stem cell fate within root and embryonic meristems. Science 347:655-660.

334 Dai X, Zhao PX. 2011. PsRNATarget: A plant small RNA target analysis server. Nucleic Acids

335 Research 39:155-159. DOI: 10.1093/nar/gkr319.

336 Damodharan S, Zhao D, Arazi T. 2016. A common miRNA160-based mechanism regulates

337 ovary patterning, floral organ abscission and lamina outgrowth in tomato. Plant Journal 86:458-

338 471. DOI: $10.1111 /$ tpj.13127.

339 Ellis CM, Nagpal P, Young JC, Hagen G, Guilfoyle TJ, Reed JW. 2005. AUXIN RESPONSE

340 FACTOR1 and AUXIN RESPONSE FACTOR2 regulate senescence and floral organ abscission

341 in Arabidopsis thaliana. Development 132:4563-4574. DOI: 10.1242/dev.02012.

342 Fleming AJ. 2006. Plant signalling: the inexorable rise of auxin. Trends in Cell Biology 16:397-

343 402. DOI: 10.1016/j.tcb.2006.06.005.

344 Guan X, Xu T, Gao S, Qi M, Wang Y, Liu X, Li T. 2014. Temporal and Spatial Distribution of

345 Auxin Response Factor Genes During Tomato Flower Abscission. Journal of Plant Growth

346 Regulation 33:317-327. DOI: 10.1007/s00344-013-9377-x.

347 Guilfoyle TJ, Hagen G. 2001. Auxin response factors. Journal of Plant Growth Regulation

348 20:281-291. DOI: 10.1007/s003440010026.

349 Guilfoyle TJ, Hagen G. 2007. Auxin response factors. Current Opinion in Plant Biology 10:453350 460. DOI: $10.1016 /$ j.pbi.2007.08.014. 
351 Guilfoyle TJ, Key JL. 1986. Auxin-regulated gene expression in higher plants. Critical Reviews

352 in Plant Sciences 4:247-276. DOI: 10.1080/07352688609382226.

353 Ha C Van, Le DT, Nishiyama R, Watanabe Y, Sulieman S, Tran UT, Mochida K, Dong N Van,

354 Yamaguchi-Shinozaki K, Shinozaki K, Tran L-SP. 2013. The auxin response factor transcription

355 factor family in soybean: genome-wide identification and expression analyses during

356 development and water stress. DNA research : an international journal for rapid publication of

357 reports on genes and genomes 20:511-24. DOI: 10.1093/dnares/dst027.

358 Hagen G, Guilfoyle T. 2002. Auxin-responsive gene expression: Genes, promoters and

359 regulatory factors. Plant Molecular Biology 49:373-385. DOI: 10.1023/A:1015207114117.

360 Jones-Rhoades MW, Bartel DP, Bartel B. 2006. MicroRNAs AND THEIR REGULATORY

361 ROLES IN PLANTS. Annual Review of Plant Biology 57:19-53. DOI:

362 10.1146/annurev.arplant.57.032905.105218.

363 Kepinski S, Leyser O. 2005. The Arabidopsis F-box protein TIR1 is an auxin receptor. Nature

364 435:446-451. DOI: 10.1038/nature03542.

365 Kim J, Harter K, Theologis A. 1997. Protein-protein interactions among the Aux/IAA proteins.

366 Proceedings of the National Academy of Sciences of the United States of America 94:11786-91.

367 DOI: 10.1073/pnas.94.22.11786.

368 Krogan NT, Ckurshumova W, Marcos D, Caragea AE, Berleth T. 2012. Deletion of MP/ARF5

369 domains III and IV reveals a requirement for Aux/IAA regulation in Arabidopsis leaf vascular

370 patterning. New Phytologist 194:391-401. DOI: 10.1111/j.1469-8137.2012.04064.x.

371 Kuang JF, Wu JY, Zhong HY, Li CQ, Chen JY, Lu WJ, Li JG. 2012. Carbohydrate stress

372 affecting fruitlet abscission and expression of genes related to auxin signal transduction pathway

373 in litchi. International Journal of Molecular Sciences 13:16084-16103. DOI:

374 10.3390/ijms131216084.

375 Le B, Nawaz MA, Rehman HM, Le T, Yang SH, Golokhvast KS, Son E, Chung G. 2016.

376 Genome-wide characterization and expression pattern of auxin response factor (ARF) gene

377 family in soybean and common bean. Genes and Genomics 38:1165-1178. DOI:

378 10.1007/s 13258-016-0462-y.

379 Li C, Wang Y, Huang X, Li J, Wang H, Li J. 2013. De novo assembly and characterization of

380 fruit transcriptome in Litchi chinensis Sonn and analysis of differentially regulated genes in fruit

381 in response to shading. BMC Genomics 14:1. DOI: 10.1186/1471-2164-14-552.

382 Li C, Wang Y, Ying P, Ma W, Li J. 2015. Genome-wide digital transcript analysis of putative 383 fruitlet abscission related genes regulated by ethephon in litchi. Frontiers in Plant Science 6:1-

384 16. DOI: 10.3389/fpls.2015.00502. 
385 Li S-B, Xie Z-Z, Hu C-G, Zhang J-Z. 2016. A Review of Auxin Response Factors (ARFs) in

386

387

388

389

390

391

392

393

394

395

396

397

398

399

400

401

402

403

404

405

406

407

408

409

410

411

412

413

414

415

416

417

418

Plants. Frontiers in plant science 7:47. DOI: 10.3389/fpls.2016.00047.

Li C, Zhang B. 2016. MicroRNAs in Control of Plant Development. Journal of Cellular Physiology 231:303-313. DOI: 10.1002/jcp.25125.

Lim PO, Lee IC, Kim J, Kim HJ, Ryu JS, Woo HR, Nam HG. 2010. Auxin response factor 2 (ARF2) plays a major role in regulating auxin-mediated leaf longevity. Journal of Experimental Botany 61:1419-1430. DOI: 10.1093/jxb/erq010.

Liscum E, Reed JW. 2002. Genetics of Aux/IAA and ARF action in plant growth and development. Plant Molecular Biology 49:387-400. DOI: 10.1023/A:1015255030047.

Liu X, Dinh TT, Li D, Shi B, Li Y, Cao X, Guo L, Pan Y, Jiao Y, Chen X. 2014a. AUXIN RESPONSE FACTOR 3 integrates the functions of AGAMOUS and APETALA2 in floral meristem determinacy. Plant Journal 80:629-641. DOI: 10.1111/tpj.12658.

Liu X, Huang J, Wang Y, Khanna K, Xie Z, Owen HA, Zhao D. 2010. The role of floral organs in carpels, an Arabidopsis loss-of-function mutation in MicroRNA160a, in organogenesis and the mechanism regulating its expression. Plant Journal 62:416-428. DOI: 10.1111/j.1365313X.2010.04164.x.

Liu Z, Miao L, Huo R, Song X, Johnson C, Kong L, Sundaresan V, Yu X. 2017. ARF2-ARF4 and ARF5 are Essential for Female and Male Gametophyte Development in Arabidopsis. Plant and Cell Physiology 59:179-189. DOI: 10.1093/pcp/pcx174.

Liu PP, Montgomery TA, Fahlgren N, Kasschau KD, Nonogaki H, Carrington JC. 2007. Repression of AUXIN RESPONSE FACTOR10 by microRNA160 is critical for seed germination and post-germination stages. Plant Journal 52:133-146. DOI: 10.1111/j.1365313X.2007.03218.x.

Liu N, Wu S, Houten J Van, Wang Y, Ding B, Fei Z, Clarke TH, Reed JW, Van Der Knaap E. 2014b. Down-regulation of AUXIN RESPONSE FACTORS 6 and 8 by microRNA 167 leads to floral development defects and female sterility in tomato. Journal of Experimental Botany 65:2507-2520. DOI: 10.1093/jxb/eru141.

Livak KJ, Schmittgen TD. 2001. Analysis of relative gene expression data using real-time quantitative PCR and the $2^{-\triangle \Delta C T}$ method. Methods 25:402-408. DOI: 10.1006/meth.2001.1262. Louie DS, Addicott FT. 1970. Applied auxin gradients and abscission in explants. Plant physiology 45:654-657.

LUO X-C, SUN M-H, XU R-R, SHU H-R, WANG J-W, ZHANG S-Z. 2014. Genomewide identification and expression analysis of the ARF gene family in apple. Journal of Genetics 93:785-797. DOI: 10.1007/s12041-014-0462-0. 
419 Ma W, Chen C, Liu Y, Zeng M, Meyers BC, Li J, Xia R. 2018. Coupling of microRNA-directed

420 phased small interfering RNA generation from long noncoding genes with alternative splicing

421 and alternative polyadenylation in small RNA-mediated gene silencing. New Phytologist

422 217:1535-1550. DOI: 10.1111/nph.14934.

423 Meir S, Philosoph-Hadas S, Sundaresan S, Selvaraj KS V., Burd S, Ophir R, Kochanek B, Reid

424 MS, Jiang C-Z, Lers A. 2010. Microarray Analysis of the Abscission-Related Transcriptome in

425 the Tomato Flower Abscission Zone in Response to Auxin Depletion. Plant Physiology

426 154:1929-1956. DOI: 10.1104/pp.110.160697.

427 Nagpal P. 2005. Auxin response factors ARF6 and ARF8 promote jasmonic acid production and

428 flower maturation. Development 132:4107-4118. DOI: 10.1242/dev.01955.

429 Nemhauser JL, Feldman LJ, Zambryski PC. 2000. Auxin and ETTIN in Arabidopsis gynoecium 430 morphogenesis. Development 127:3877-3888.

431 Okushima Y, Overvoorde PJ, Arima K, Alonso JM, Chan A, Chang C, Ecker JR, Hughes B, Lui

432 A, Nguyen D, Onodera C, Quach H, Smith A. 2005. Functional Genomic Analysis of the

433 AUXIN RESPONSE FACTOR Gene Family Members in Arabidopsis thaliana. The Plant Cell

434 17:444-463. DOI: 10.1105/tpc.104.028316.2.

435 Pattison RJ, Csukasi F, Catalá C. 2014. Mechanisms regulating auxin action during fruit

436 development. Physiologia Plantarum 151:62-72. DOI: 10.1111/ppl.12142.

437 Peng G, Wu J, Lu W, Li J. 2013. A polygalacturonase gene clustered into clade E involved in

438 lychee fruitlet abscission. Scientia Horticulturae 150:244-250. DOI:

439 10.1016/j.scienta.2012.10.029.

440 Peng M, Ying P, Liu X, Li C, Xia R, Li J, Zhao M. 2017. Genome-Wide Identification of

441 Histone Modifiers and Their Expression Patterns during Fruit Abscission in Litchi. Frontiers in

442 Plant Science 8:1-16. DOI: 10.3389/fpls.2017.00639.

443 Piya S, Shrestha SK, Binder B, Stewart CN, Hewezi T. 2014. Protein-protein interaction and

444 gene co-expression maps of ARFs and Aux/IAAs in Arabidopsis. Frontiers in Plant Science 5:1-

445 9. DOI: 10.3389/fpls.2014.00744.

446 Rademacher EH, Lokerse AS, Schlereth A, Llavata-Peris CI, Bayer M, Kientz M, FreireRios A,

447 Borst JW, Lukowitz W, Jürgens G, Weijers D. 2012. Different Auxin Response Machineries

448 Control Distinct Cell Fates in the Early Plant Embryo. Developmental Cell 22:211-222. DOI:

449 10.1016/j.devcel.2011.10.026.

450 Ren Z, Liu R, Gu W, Dong X. 2017. The Solanum lycopersicum auxin response factor SlARF2

451 participates in regulating lateral root formation and flower organ senescence. Plant Science

452 256:103-111. DOI: 10.1016/j.plantsci.2016.12.008. 
$453 \mathrm{Ru}$ P, Xu L, Ma H, Huang H. 2006. Plant fertility defects induced by the enhanced expression of 454 microRNA167. Cell Research 16:457-465. DOI: 10.1038/sj.cr.7310057.

455 Stern RA, Gazit S. 2000. Application of the polyamine putrescine increased yield, of "Mauritius" 456 litchi (Litchi chinensis Sonn.). Journal of Horticultural Science and Biotechnology 75:612-614.

457 DOI: $10.1080 / 14620316.2000 .11511295$.

458 Sundberg E, Østergaard L. 2009. Distinct and dynamic auxin activities during reproductive 459 development. Cold Spring Harbor perspectives in biology 1:1-15. DOI:

460 10.1101/cshperspect.a001628.

461 Tiwari SB, Hagen G, Guilfoyle T. 2003. The Roles of Auxin Response Factor Domains in 462 Auxin-Responsive Transcription. Plant Cell 15:533-543. DOI: 10.1105/tpc.008417.All.

463 Wagner GP, Kin K, Lynch VJ. 2012. Measurement of mRNA abundance using RNA-seq data:

464 RPKM measure is inconsistent among samples. Theory in Biosciences 131:281-285. DOI: 465 10.1007/s12064-012-0162-3.

466 Wang Y, Deng D, Shi Y, Miao N, Bian Y, Yin Z. 2012. Diversification, phylogeny and 467 evolution of auxin response factor (ARF) family: Insights gained from analyzing maize ARF 468 genes. Molecular Biology Reports 39:2401-2415. DOI: 10.1007/s11033-011-0991-z.

469 Wang D, Pei K, Fu Y, Sun Z, Li S, Liu H, Tang K, Han B, Tao Y. 2007. Genome-wide analysis 470 of the auxin response factors (ARF) gene family in rice (Oryza sativa). Gene 394:13-24. DOI: 471 S0378-1119(07)00032-7 [pii] \r10.1016/j.gene.2007.01.006.

472 Woodward AW, Bartel B. 2005. Auxin: Regulation, action, and interaction. Annals of Botany 473 95:707-735. DOI: 10.1093/aob/mci083.

474 Wu M-F, Tian Q, Reed JW. 2006. Arabidopsis microRNA167 controls patterns of ARF6 and 475 ARF8 expression, and regulates both female and male reproduction. Development 133:4211476 4218. DOI: $10.1242 / \mathrm{dev} .02602$.

477 Xia R, Xu J, Meyers BC. 2017. The Emergence, Evolution, and Diversification of the miR390-

478 TAS3-ARF Pathway in Land Plants. The Plant Cell 29:tpc.00185.2017. DOI:

$47910.1105 /$ tpc. 17.00185.

480 Xie RJ, Deng L, Jing L, He SL, Ma YT, Yi SL, Zheng YQ, Zheng L. 2013. Recent advances in 481 molecular events of fruit abscission. 57:201-209. DOI: 10.1007//s10535-012-0282-0.

482 Xie R, Pang S, Ma Y, Deng L, He S, Yi S, Lv Q, Zheng Y. 2015. The ARF, AUX/IAA and GH3 483 gene families in citrus: genome-wide identification and expression analysis during fruitlet drop 484 from abscission zone A. Molecular Genetics and Genomics 290:2089-2105. DOI:

485 10.1007/s00438-015-1063-1.

486 Xing H, Pudake RN, Guo G, Xing G, Hu Z, Zhang Y, Sun Q, Ni Z. 2011. Genome-wide 
487 identification and expression profiling of auxin response factor (ARF) gene family in maize.

488 BMC Genomics 12:178. DOI: 10.1186/1471-2164-12-178.

489 Xu T, Wang Y, Liu X, Lv S, Feng C, Qi M, Li T. 2015. Small RNA and degradome sequencing

490 reveals microRNAs and their targets involved in tomato pedicel abscission. Planta 242:963-984.

491 DOI: 10.1007/s00425-015-2318-0.

492 Yuan R. 1988. Litchi Fruit Abscission: its Patterns, Effect of Shading and Relation to

493 Endogenous Abscisic Acid 1. 36:281-292.

494 Zhong H-Y, Chen J-W, Li C-Q, Chen L, Wu J-Y, Chen J-Y, Lu W-J, Li J-G. 2011. Selection of

495 reliable reference genes for expression studies by reverse transcription quantitative real-time

496 PCR in litchi under different experimental conditions. Plant cell reports 30:641-53. DOI:

497 10.1007/s00299-010-0992-8.

498 Zouine M, Fu Y, Chateigner-Boutin AL, Mila I, Frasse P, Wang H, Audran C, Roustan JP,

499 Bouzayen M. 2014. Characterization of the tomato ARF gene family uncovers a multi-levels

500 post-transcriptional regulation including alternative splicing. PLOS ONE 9:1-12. DOI:

501 10.1371/journal.pone.0084203.

502 
Figure 1

Phylogenetic analysis of ARFs from litchi, rice, Arabidopsis and tomato.

The phylogenetic tree was generated using the Maximum Likelihood method with the JTT matrix-based model (Kumar, Stecher \& Tamura, 2016) and the bootstrap test was carried out with 1000 bootstrap replicates. Numbers on the nodes indicate the credibility values of each clay. Three subgroups were shown as Class I, II, and III.

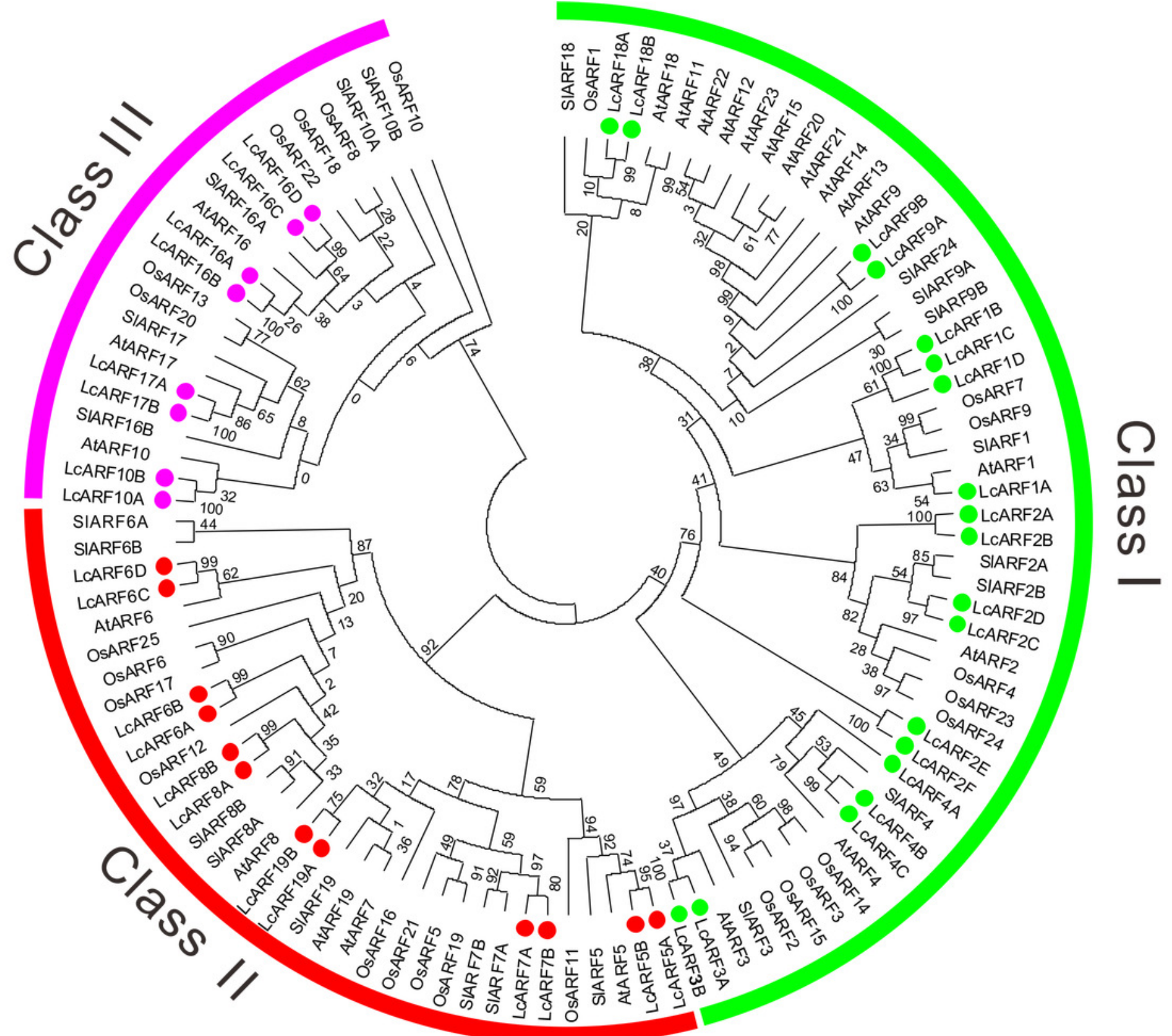




\section{Figure 2}

Phylogenetic relationship, exon-intron structure, conserved domains analyses of LCARFs.

(A) Phylogenetic relationship among the litchi ARF proteins. The unrooted tree was generated using the maximum likelihood method by JTT matrix-based model. The reliability was assessed using 1000 bootstrap replicates. Three clusters are labeled as Class I, Class II and Class III. (B) Exon- intron structure and conserved domains of LcARFs. Information of exon, intron, and functional domain was obtained from model gene annotation and results of NCBI CDD search and visualized by TBtools. B3: B3 DNA-binding domain; Auxin-resp: ARF domain; AUX_IAA: C-terminal dimerization domain. Lengths of exons and introns and domains of each LCARF protein were exhibited proportionally. 
A

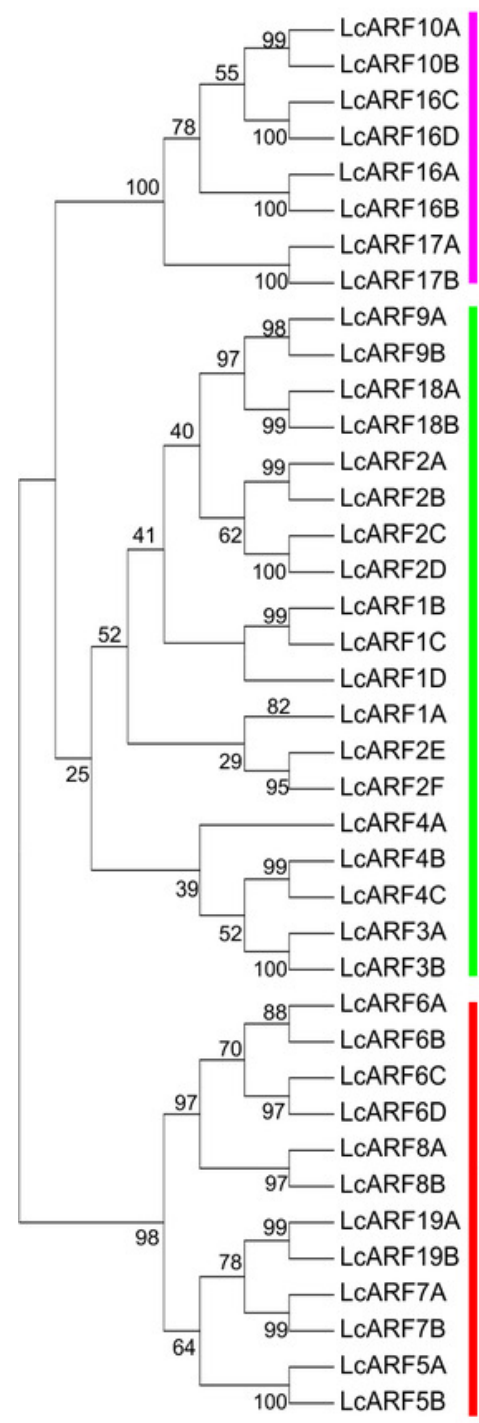

B

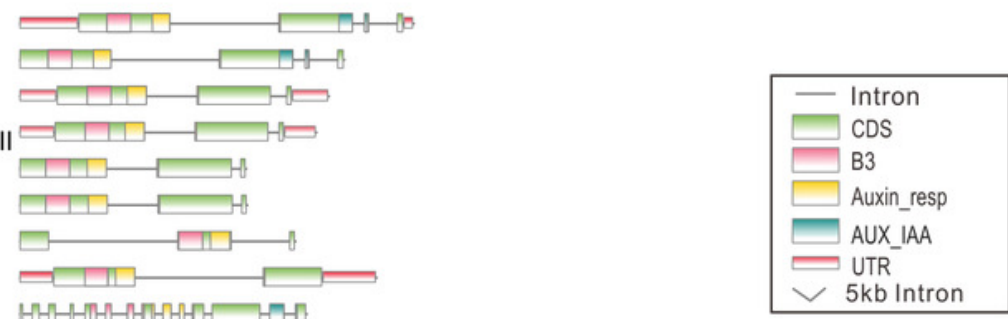

HНH

$\longrightarrow \mathrm{HHH}$

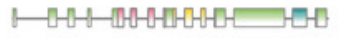

$\mapsto \mathbb{H}-\mathbb{H}-\mathrm{HH}=-\mathrm{HE}$

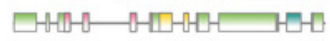

$\Longrightarrow$

MHHIH-HI- OHE-

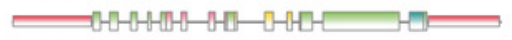

Class I

औHњ

난, 1

$\longmapsto \longrightarrow$

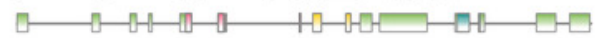

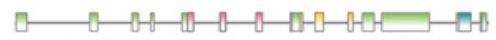

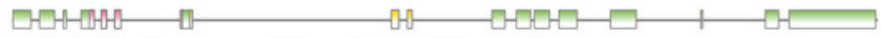

$\mathrm{OH}-\mathrm{HH}-\mathrm{IL}$

매느믈

टण-

$\square \mathrm{C}-\mathrm{MH} H \mathrm{H}-\mathrm{H}$

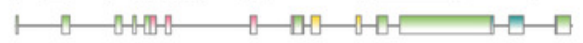

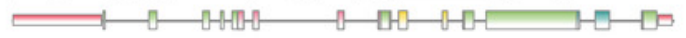

I-

Class II

$\longmapsto$

НH,

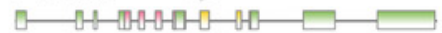

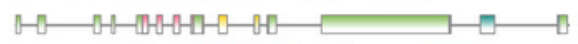

$\longmapsto$

ए

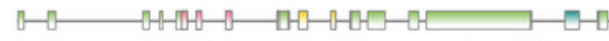

H-

$5^{\prime}$

$0 \mathrm{~kb}$

$2.5 \mathrm{~kb}$

$5 \mathrm{~kb}$

$7.5 \mathrm{~kb}$

$10 \mathrm{~kb}$

$12.5 \mathrm{~kb}$ 
Figure 3

LCARFs targeted by some LcmiRNAs.

miR160 targets $L C A R F 10 / 16 / 17$, miR167 targets $L C A R F 6 / 8$ and miRN43 targets LCARF9. Additionally, the LCTAS3 was targeted by miR390 and then triggered the production of tasiARF which target $L C A R F 2 E / 2 F / 4 B / 4 C / 3 A / 3 B$. 


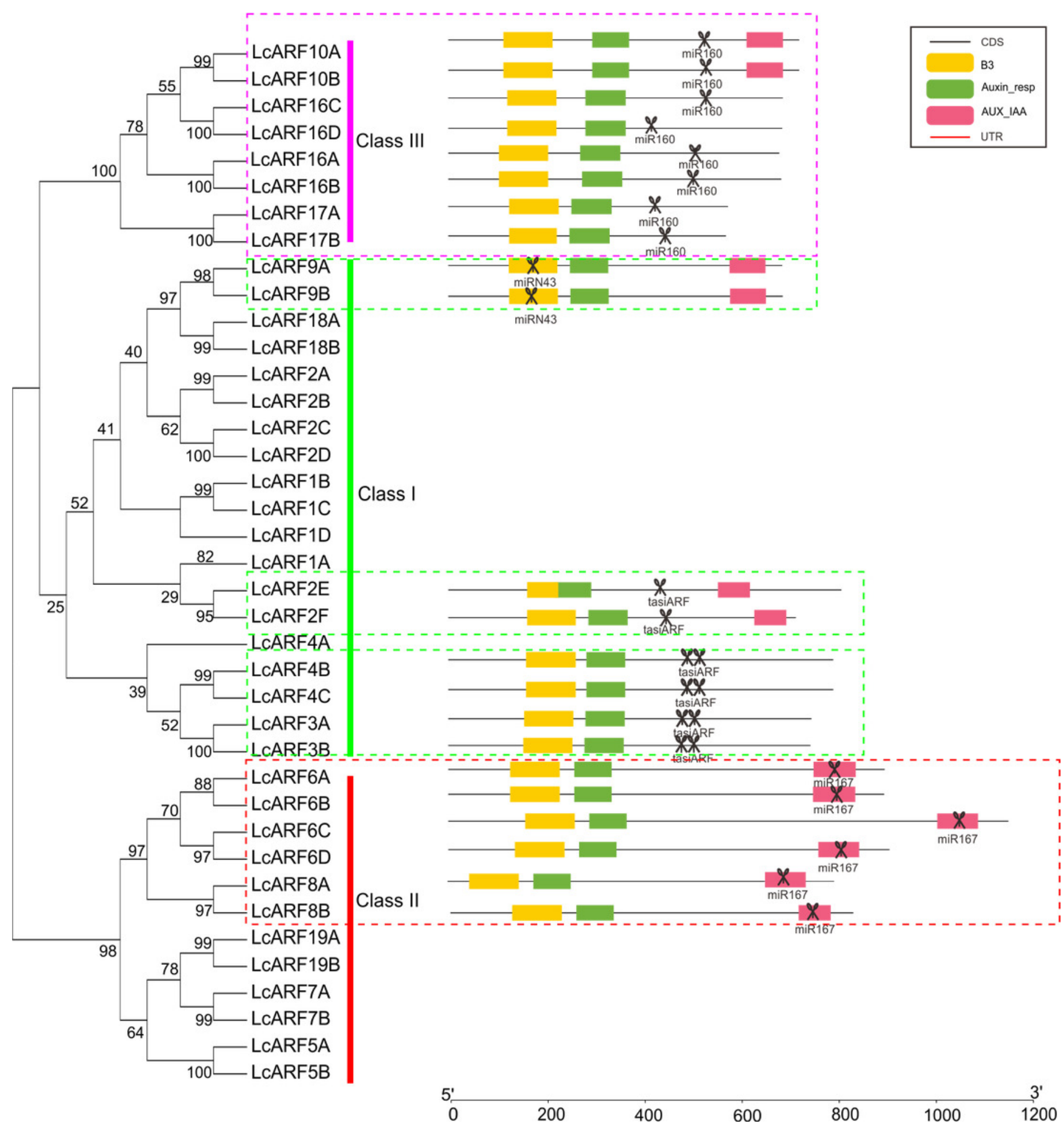




\section{Figure 4}

Expression Profiles of LCARF Genes in various tissues of 'FZX' by qRT-PCR.

A) Expression of all LCARFs in different tissues. The heatmap was generated based on the relative expression values of 39 LCARFs obtained by qRT-PCR in seven different tissues and organs. Red and blue were represented relatively high and lower expression ( $\log _{2}$ ratio), respectively. Every sample has three biological replicates. YF: young fruit ( 25 days after fertilization), FF: female flower, MF: male flower, USF: undeterminated sex flowers, ML: mature leaves, YL: young leaves, FBS: fruit-bearing shoots. B) Relative abundance of LCARFs significantly expressed in FF. Data from three independent biological replicates are shown with standard deviation (SD). Asterisks on the top of bars indicate significant differences as determined by Student's t-test (*P, 0.05). 
A

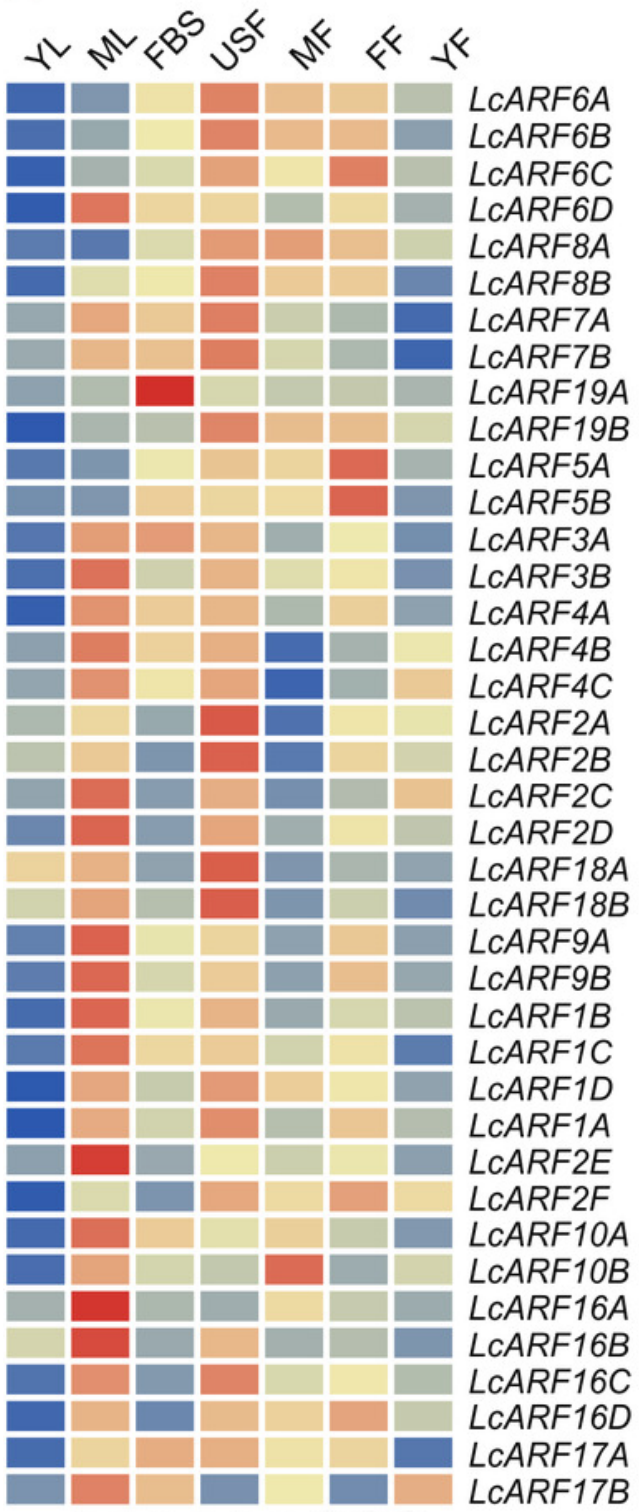

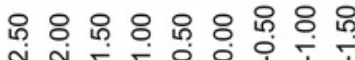

B
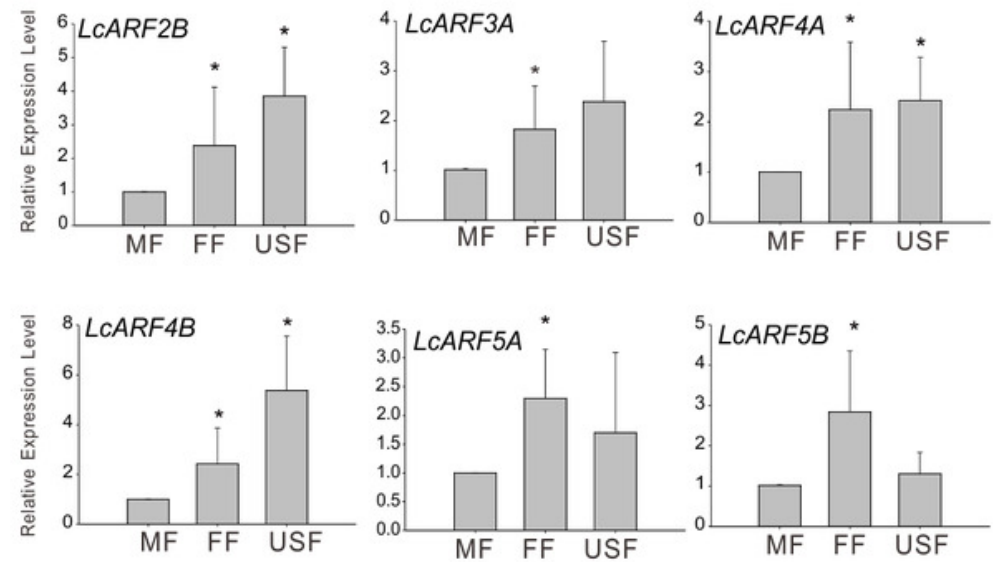

Class I
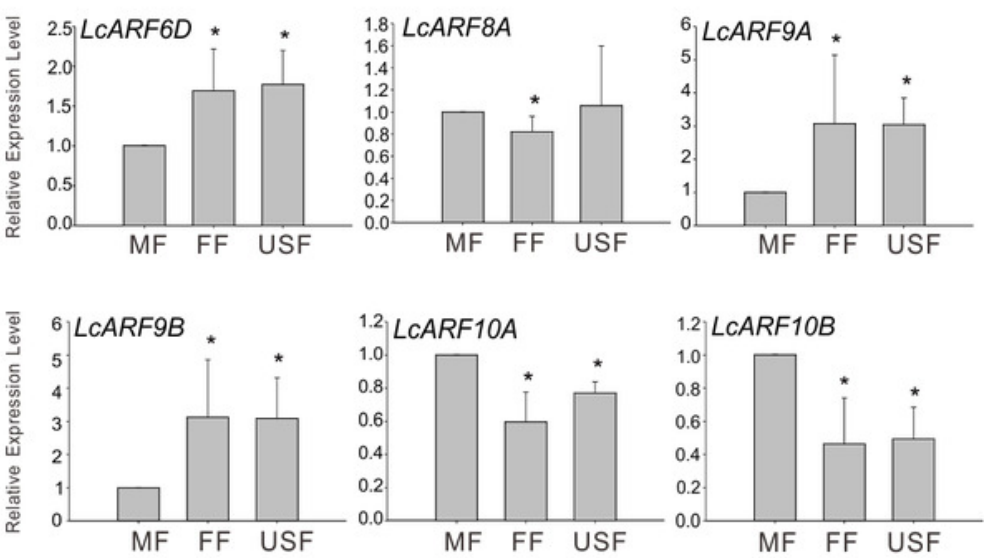

Class III

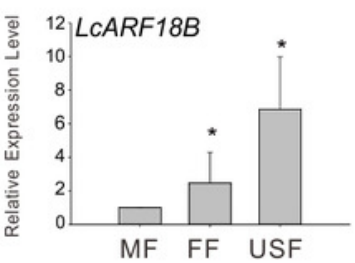




\section{Figure 5}

Expression Profiles of LCARF Genes in Response to ETH, GPD, and GPDD treatments.

Fruit-bearing shoots of ' $F Z X$ ' litchi were obtained at 25 days after anthesis and then carried on ETH, GPD, and GPDD treatment from 0, 1, 2 and 3 days, respectively. CK: control; ETH:

treated by ethylene; GPD: girdling plus defoliation; GPDD: dipping in 2, 4-D after GPD treatment. The heatmap was created based on the TPM values of LCARFs from the transcriptome data. In the heatmap, red and blue were represented higher and lower expression ( $\log _{2}$ ratio), respectively. Heatmap and hierarchical clustering were performed by average linage (default) method. 


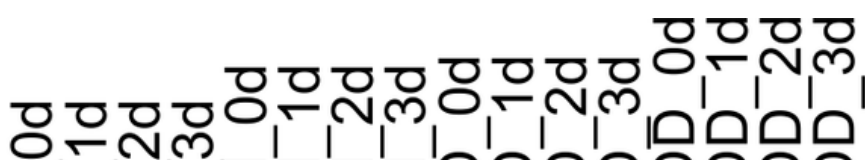

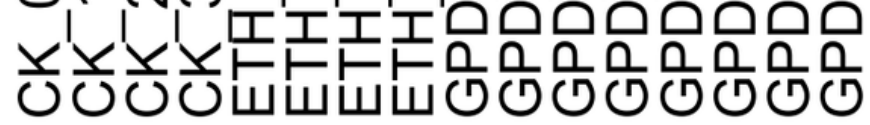

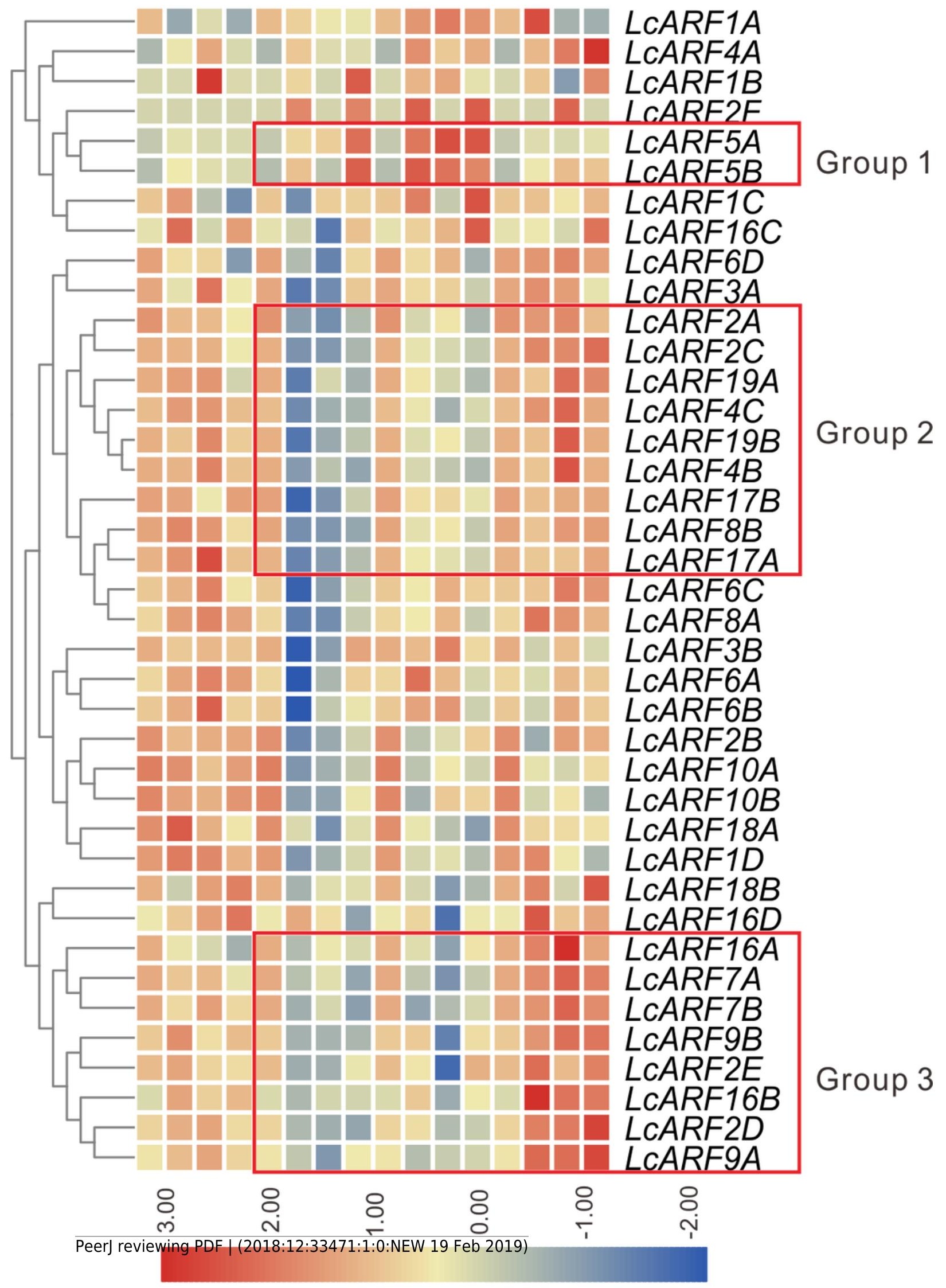

